University of Louisville

ThinkIR: The University of Louisville's Institutional Repository

\title{
An investigation into the adiponectin receptor levels in cardiac, liver and skeletal muscle of mature rats.
}

Jacqueline Sippel

University of Louisville

Follow this and additional works at: https://ir.library.louisville.edu/honors

Part of the Biology Commons

\section{Recommended Citation}

Sippel, Jacqueline, "An investigation into the adiponectin receptor levels in cardiac, liver and skeletal muscle of mature rats." (2016). College of Arts \& Sciences Senior Honors Theses. Paper 125.

http://doi.org/10.18297/honors/125

This Senior Honors Thesis is brought to you for free and open access by the College of Arts \& Sciences at ThinkIR: The University of Louisville's Institutional Repository. It has been accepted for inclusion in College of Arts \& Sciences Senior Honors Theses by an authorized administrator of ThinkIR: The University of Louisville's Institutional Repository. This title appears here courtesy of the author, who has retained all other copyrights. For more information, please contact thinkir@louisville.edu. 
An Investigation into the Adiponectin Receptor Levels in Cardiac, Liver and Skeletal Muscle of Mature Rats

\author{
By \\ Jacqueline Sippel
}

Submitted in partial fulfillment of the requirements for Graduation summa cum laude and

for Graduation with Honors from the Department of Biology

University of Louisville

March, 2016 


\section{Table of Contents}

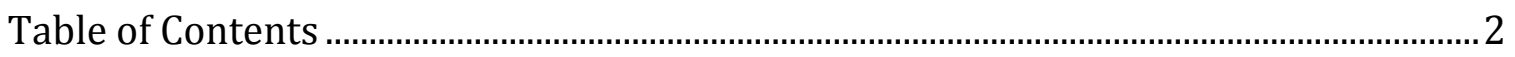

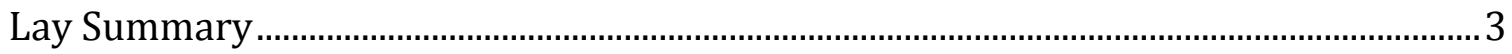

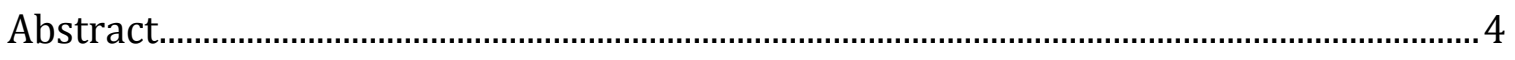

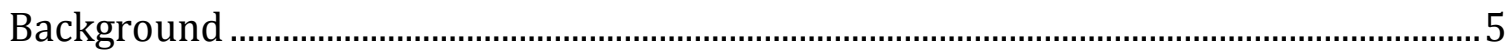



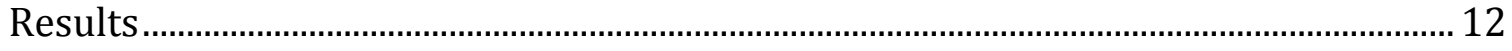

Protein assay to determine total protein content …………………………………... 12

ELISA to determine the content of AdipoR1 and AdipoR2 ……………………......... 13

Tissue content of AdipoR1 and AdipoR2 by sex ........................................................ 18

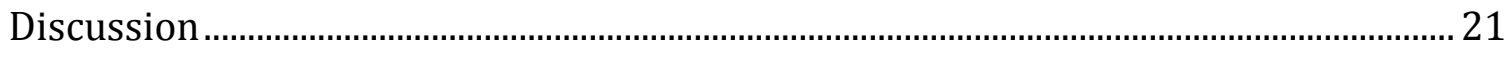

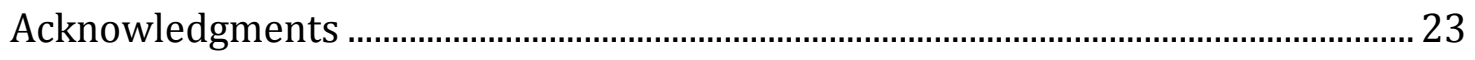

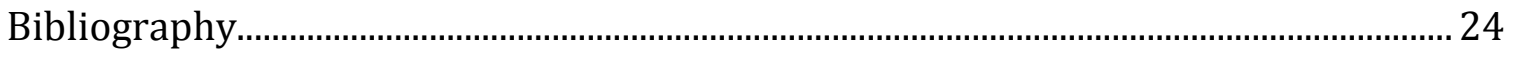




\section{Lay Summary}

Both obesity and diabetes are domestic and international health problems.

Adiponectin is a protein secreted from fat cells and muscle tissue, which influences the metabolism of sugars and fats. For this reason, Adiponectin is a primary target for therapeutic treatment of these metabolic diseases. The aim of this study is to determine the levels of AdipoR1 and AdipoR2 (the receptors for adiponectin) within cardiac, skeletal muscle, and liver tissue samples of mature non-obese rats in order to determine if the rat can be used as a model for studies of adiponectin. The data from the current study support previous work on the relative levels of AdipoR1 in muscle and liver tissues; however, the data from the current study contradict that of previous reports, which had suggested that liver had a higher level of AdipoR2 than muscle tissue, indicating that further investigation and confirmation is necessary to determine if the rat is a robust model for studies of adiponectin receptors. 


\section{Abstract}

Both obesity and diabetes are domestic and international health problems. Adiponectin is a protein secreted from adipocytes and other tissues such as muscle which influences carbohydrate and lipid metabolism. For this reason, Adiponectin is a primary target for therapeutic treatment of metabolic diseases. Adiponectin interacts with receptors termed AdipoR1 and AdipoR2, which are structurally similar to G protein-coupled receptors except with a reverse membrane topology. The aim of this study is to determine the levels of AdipoR1 and AdipoR2 within cardiac, skeletal, and liver tissue samples of mature non-obese rats. The objective of determining potential differences in the tissue levels of these receptors and determining any gender differences will be used to establish whether or not the rat could make a viable model for further study in adiponectin related research. Samples of tissue were homogenized, the lysate centrifuged to produce a fluid supernatant, and the supernatants' protein content analyzed. AdipoR1 and AdipoR2 levels were determined by ELISA (NeoBiolab, Boston, MA). The data supports a difference $(p \leq 0.02)$ in AdipoR1 levels between the liver and muscle tissues (gastrocnemius, soleus, and heart), as well as between the genders for the gastrocnemius and liver. The levels of AdipoR2 in the liver were also significantly lower than in muscles (heart and gastrocnemius), with significant differences ( $\mathrm{p} \leq$ 0.01 ) between the genders for liver. The data from the current study supports previous work on the relative levels of AdipoR1 in muscle and liver, but does not identify any difference between muscle types. In addition, gender differences in the levels of AdipoR1 in gastrocnemius and liver are novel observations. However, the data from the current study contradicts that of previous reports, which had suggested that liver had a higher level of AdipoR2 than muscle tissue, indicating that further investigation and confirmation is necessary. 


\section{Background}

Obesity and diabetes are local and international health problems that have become more prevalent in recent years, with over one third of Americans over the age of twenty classified as obese (15). Diseases such as type 2 diabetes (T2D), cardiovascular diseases, and some cancers have been linked to obesity $(4,7,11)$. Due to the severity of these conditions, the signaling molecules involved in metabolism need to be further investigated. Adiponectin is one of the most prominent plasma adipokines or adipose-derived cell signaling proteins $(1,4,6,7,14)$. It is a plasma protein with insulin-sensitizing properties in addition to anti-inflammatory, and anti-plaque forming properties $(1,3,4,5,7,9,11,14)$. Levels of circulating adiponectin were found to be lower in obese, insulin resistant, and type 2 diabetic humans and animal models of these conditions $(1,4,5,9,11)$. Adiponectin has also been shown to influence many other functions including decreasing both inflammation and cellular apoptosis $(2,4,7,11)$.

The 30-kDa adiponectin monomer (Figure 1) consists of an amino-terminal domain followed by a collagen-like domain and a carboxyl-terminal globular domain $(1,7,14)$. The amino acid-terminal domain contains a 17 -amino acid portion that serves as a signal sequence to propel it into the secretory pathway (22). The primary amino acid sequence of the adiponectin gene is highly conserved, at greater than $80 \%$ homology, among several species including humans, mice and rats (17). There are three major forms of circulating adiponectin (Figure 1): a low-molecularweight (LMW) trimer, a middle-molecular-weight (MMW) hexamer, and a high- 
molecular-weight (HMW) 12- 18-mer $(7,8,9,12)$.

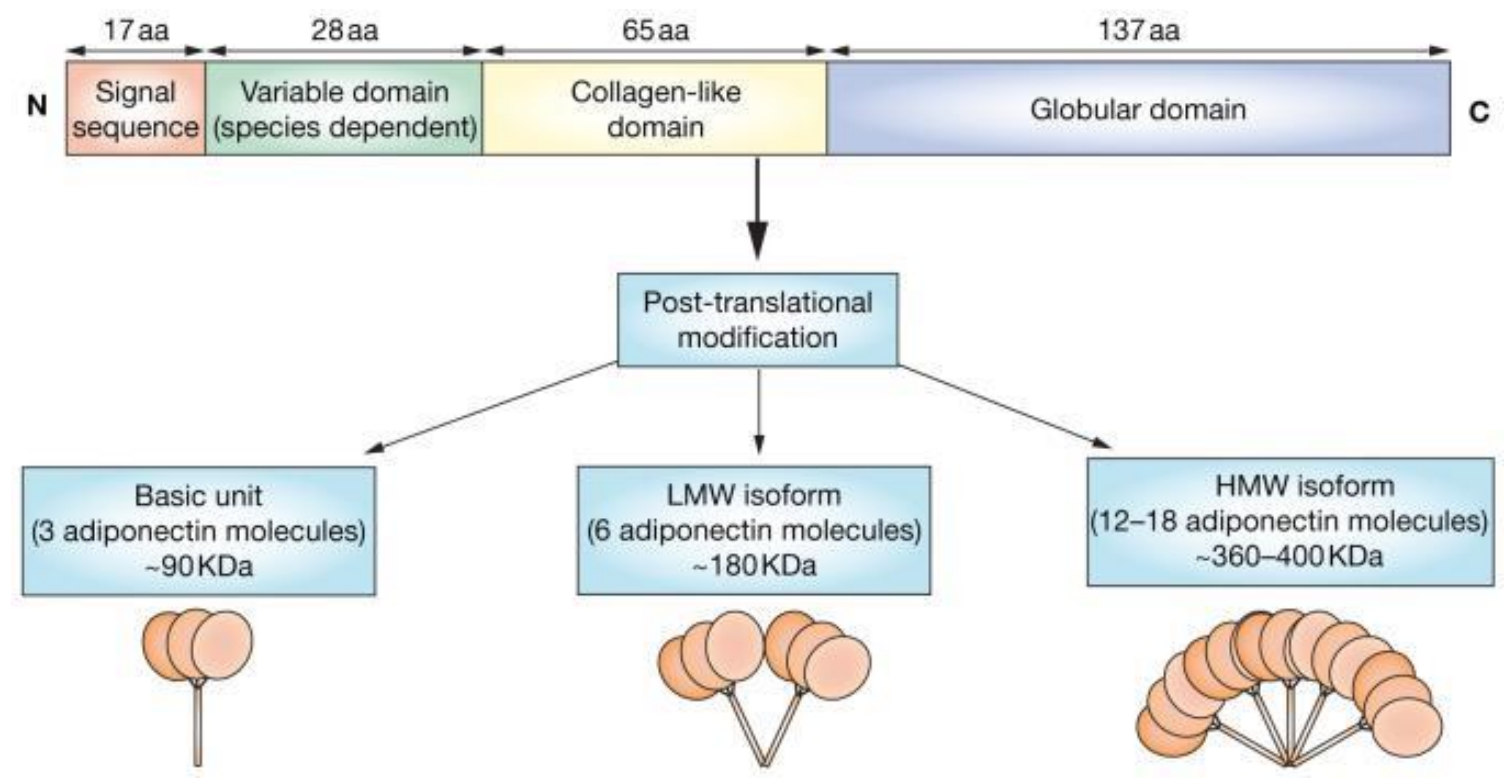

Figure 1: Structure of adiponectin. Adiponectin contains three domains and a signal sequence. After post-translational modifications such as glycosylation, adiponectin is secreted from the cell as the basic trimer unit, a low-molecular weight (LMW) isoform, or a high molecular weight (HMW) isoform. Other abbreviations: amino-acids (aa), carboxy-terminal (C), amino-terminal (N) (22).

The LMW trimer is the basic building block of adiponectin and is formed via the hydrophobic interactions within the globular heads and stabilized by the noncovalent interactions of the collagenous domains (17). The MMW complex, which is not shown in Figure 1, is formed via the disulfide bond of two cysteine residues located in the N-terminal variable region $(11,17,21)$. The HMW complex is formed in a similar way, with the N-terminal region associations allowing for disulfide bonds between the cysteine residues $(17,21)$. The HMW is the most active form, making it the most relevant to studies of insulin sensitivity and diabetes $(7,9,11,14)$. It is the ratio of HMW to total adiponectin, not the total adiponectin levels, that 
correlates with levels of insulin sensitivity $(17,19)$. Further, the elevation of LMW and MMW in cerebrospinal fluid has been shown to aggravate obesity by not only increasing food intake, but also by suppressing energy expenditure (17). This suggests that there may be adiponectin targets in the central nervous system that can be manipulated for therapy.

A sexual dimorphism of adiponectin concentration has been reported in both humans and rodents, with males reported to have significantly lower plasma levels of total adiponectin than females, which has been attributed primarily to the inhibitory actions of testosterone on adiponectin secretion (17). It has been noted that neither castration nor testosterone treatment interferes with the abundance of adiponectin mRNA, suggesting that the adiponectin levels in males are being affected by preferential secretion of the LMW and MMW over the HMW (17). This selective secretion therefore implies that there are at least two different secretory mechanisms, which allows testosterone to selectively inhibit the release of HWM adiponectin (17). A study from Bogan and Lodish (18) showed two distinct pathways exist, which allows for constitutive as well as regulated secretion.

Adiponectin significantly affects insulin sensitivity through direct action on liver and muscle $(1,4,5,11,19)$. The biological effects depend not only on the circulating concentration of adiponectin, but also on the expression levels and functionality of its receptors $(1,4,7)$. Reduced levels of circulating adiponectin and its receptors can be caused by genetic factors within the adiponectin gene itself as well as environmental factors that cause obesity, such as a high fat diet $(3,4,14)$. It 
has also been found that plasma adiponectin levels are inversely associated with obesity, diabetes, glucose, lipid, and insulin resistance, whereas the receptor levels are positively associated with these disease states (10).

Adiponectin regulates skeletal muscle energy metabolism by stimulating free fatty acid (FFA) oxidation by mitochondria as well as glucose uptake via enhanced GLUT4 translocation to the plasma membrane $(1,3,4,7)$. Impaired adiponectin levels have been linked with T2D $(3,5,7)$, where subjects with high concentrations of circulating adiponectin were $40 \%$ less likely to develop T2D, indicating that adiponectin could be used as a predictor for future development of T2D (3). It has also been shown that circulating adiponectin levels are decreased in rodents fed high fat diets, while the addition of adiponectin via injection results in an improvement in insulin resistance $(4,7)$.

The pleiotropic effects of adiponectin are mediated by two receptors (Figure 2) named AdipoR1 and AdipoR2 (1,4,5,7,11). Both are the major receptors for adiponectin in vivo and play an important role in glucose metabolism and insulin sensitivity $(1,4,7)$. AdipoR1 is ubiquitously expressed, but is predominately found in skeletal muscle, whereas AdipoR2 is reported to be most abundant in liver $(1,3,4,5,7,10,11)$. AdipoR1/R2 have also been discovered on pancreatic $\beta$ cells suggesting they may also play a role in insulin secretion (19). AdipoR1/R2 doubleknockout mice have conclusively shown that without these receptors, the major adiponectin-induced metabolic effects (such as increased insulin sensitivity) were completely obliterated (20). 
The two receptors are approximately $67 \%$ homologous in their amino acid sequence $(1,4,7)$. The receptors consist of a seven-transmembrane domain similar to G-protein-coupled receptors except for the C-terminus and N-terminus being reversed so that the adiponectin receptors' C-terminus is extracellular and the $\mathrm{N}$ terminus is cytosolic $(1,4,7,19)$, which can be seen in Figure 2. AdipoR1 and AdipoR2 also differ from G-protein- coupled receptors in their downstream signaling mechanisms $(1,20)$.

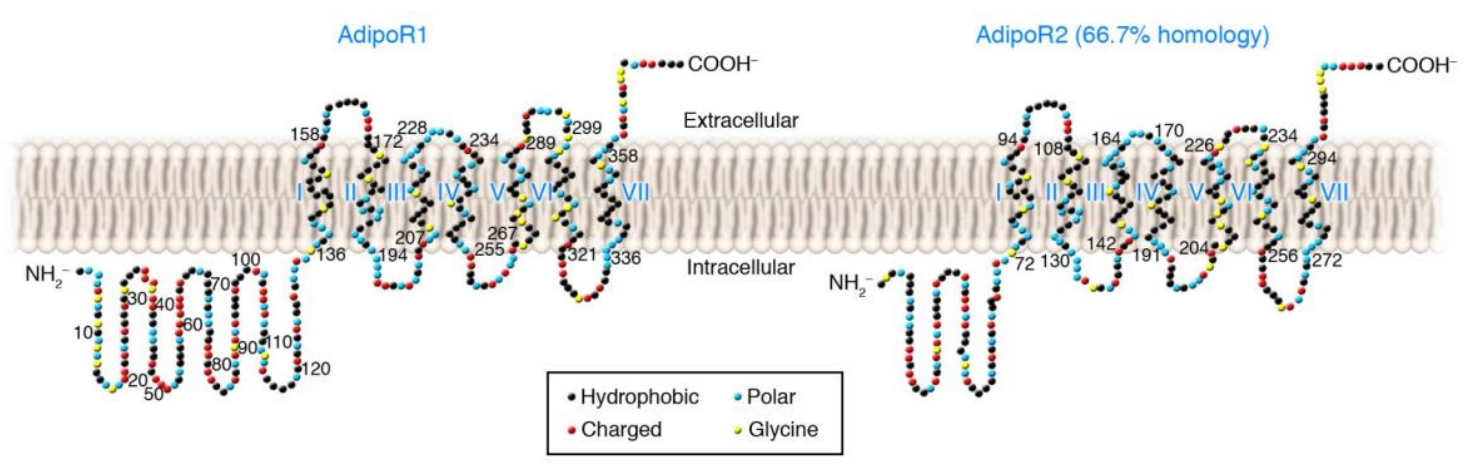

Figure 2: AdipoR1 and AdipoR2 structure. AdipoR1/R2 share a 67\% homology in their amino acid sequence and consist of seven transmembrane domains. The C-terminus is located in the extracellular space, while the $\mathrm{N}$-terminus is located in the intracellular space (19).

AdipoR1 has a high affinity for globular Adiponectin (gAd), which consists of just the c-terminal globular domain, and a low affinity for the full adiponectin (fAd) $(1,3,4,5,7,10,11)$. AdipoR2 has an intermediate affinity for both the fAd and gAd $(1,4,5,7,10,11)$. Studies suggest that AdipoR1 plays a crucial role in mitochondrial dysfunction and insulin resistance in muscle and thus may be a potential therapeutic target $(1,3,4)$. AdipoR1 and AdipoR2 were significantly decreased in muscles and adipose tissue of insulin-resistant subjects, compared to control 
subjects $(3,5,19)$. It has been suggested that AdipoR1 directly affects the expression of genes that code for hepatic gluconeogenic enzymes therefore decreasing endogenous glucose production (20). AdipoR2, unlike AdipoR1, has been shown to increase glucose uptake, but it has no effect on the gene expression involved with gluconeogenesis (20). AdipoR2 has also been shown to reduce inflammation and oxidative stress, which may explain the improvement in liver insulin resistance (20).

The topic of this thesis is determination of levels of the two main receptors for adiponectin, which warrant further study as they may prove to be a valuable tool for predicting diabetes and other obesity-related diseases as well as being a focus for therapeutic intervention. The aim of this thesis is to assess the level of AdipoR1/R2 in multiple tissue types for comparison and to consider if the rat will make a viable model for the study of the role of adiponectin in diabetes and obesity. I hypothesize that the levels of AdipoR1/R2 will be comparable to those levels reported in other organisms, and that the rat will indeed be a practical model for further testing.

\section{Methods}

Tissue samples, obtained from mature male rats utilized by the Price Institute of Surgical Research (Louisville, KY) and mature female sentinel rats from the Research Resources Center (University of Louisville), were frozen in liquid nitrogen and stored at $-80^{\circ} \mathrm{C}$ until used. Milligram quantities of frozen tissue samples were weighed using a Mettler AE 100 (Mettler-Toledo Ltd., Leicester, UK) 
and homogenized with a Micro-vial Homogenizer (Wilmad LabGlass, Elk Grove Village, IL) at $4^{\circ} \mathrm{C}$ in $0.6 \mathrm{ml}$ of cold buffer (20 mM Tris- $\mathrm{HCl}$, pH7.5, $50 \mathrm{mM} 2$ mercaptoethanol, 5 mM EGTA, 2 mM EDTA, 1mM phenylmethylsulfonyl fluoride, 10 mM sodium fluoride, $25 \mu \mathrm{g} / \mathrm{ml}$ leupeptin, $2 \mu \mathrm{g} / \mathrm{ml}$ aprotinin) (13). The homogenized samples were centrifuged at $10,000 \mathrm{RPM}(8161 \mathrm{~g})$ for ten minutes at $4^{\circ} \mathrm{C}$. The supernatants were collected and stored at $-80^{\circ} \mathrm{C}$ until analyses were conducted (13).

The total protein content of each sample was measured in duplicate using a protein assay (16) with bovine serum albumin (Pierce, Rockford, IL) as a standard. The absorbance was measured on a Milton Roy Spectronic 601 at $650 \mathrm{~nm}$ and the duplicate readings were averaged to form a standard curve on Microsoft Excel along with the line of best fit. The tissue samples were thawed on ice and each of the tissue types (liver, gastrocnemius, soleus, and heart) were run (with $3 \mu \mathrm{L}$ of sample and $997 \mu \mathrm{L}$ of DI water) and the absorbance read. Using the line of best fit from the standard curve, the amount of total protein was determined for all samples.

Competitive ELISAs (NeoBiolab, Cambridge, MA) were run to assess the total amount of AdipoR1/R2. First, all kit components and samples were brought up to room temperature. Next, $200 \mu \mathrm{L}$ of each sample was pipetted into labeled Eppendorf tubes and $20 \mu \mathrm{L}$ of balance solution was added and all tubes vortexed. $100 \mu \mathrm{L}$ of the samples as well as the standards were pipetted into the pre-blocked wells in duplicate in a 96 well plate. After all wells were loaded, $50 \mu \mathrm{L}$ of enzyme conjugate was added to each well. The plate was then incubated for one hour in humid chamber kept at $37^{\circ} \mathrm{C}$. 
Once the incubation was complete, the plate was washed five times with 400 $\mu \mathrm{L}$ of wash solution, which had been diluted from $100 \mathrm{x}$ concentration to $1 \mathrm{x}$ concentration. After each wash, the plate was inverted to remove the liquid and blotted dry. Next, $50 \mu \mathrm{L}$ of Substrate A was added to each well, followed by the addition of $50 \mu \mathrm{L}$ of Substrate B. The plate was then covered in foil and incubated again, this time for fifteen minutes at room temperature in the dark. After fifteen minutes, $50 \mu \mathrm{L}$ of the Stop Solution was added to each well and the plate was immediately read at $450 \mathrm{~nm}$ using an ELX 808 Ultra Microplate Reader. All samples, including the standards and blanks were run in duplicate and the plate reader values averaged in order to determine the concentration of AdipoR1/R2 using the standard curve. Finally, data analyses (ANOVA, t-test, significant difference $[\mathrm{p}<0.05])$ were performed using Microsoft Excel and StatsPlus.

\section{Results}

\section{Protein assay to determine total protein content}

In order to determine the amount of total protein in each tissue homogenate for use in expressing total receptor levels, protein assays were performed as described in the methods section. Using a standard curve generated for each assay (an example of one of the standard curves can be seen in Figure 3), the average amount of protein per milliliter of sample was determined for each tissue homogenate. The standard curves all had an $\mathrm{R}^{2}$ value of at least 0.99 , meaning the standards fell very close to the line of best fit. The equations for the line of best fit 
were then rearranged to solve for " $\mathrm{x}$ "; then that number was corrected for dilution in order to calculate $\mathrm{mg}$ of protein per $\mathrm{mL}$ of homogenized sample. The average amount of protein per mL was then found for the genders for each tissue type, along with the standard deviation. At least six animals (three from each gender) for each tissue were measured in duplicate.

\section{Protein Assay for AdipoR2}

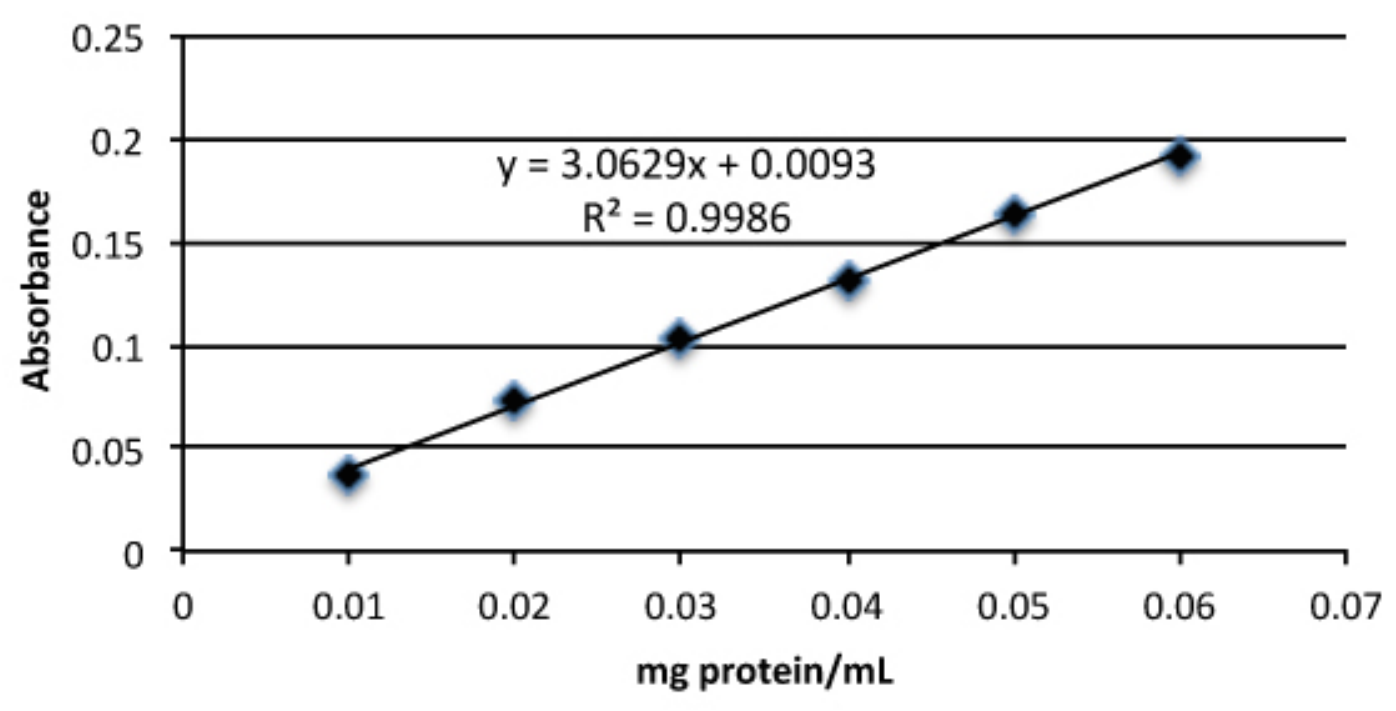

Figure 3: Typical Protein assay results for Liver, Heart, and Gastrocnemius. A standard curve for both male and female samples of the liver, heart, and gastrocnemius with an $R^{2}$ value of 0.99 . The other standard curves generated look similar to this and have an $R^{2}$ value of at least 0.99 as well. At least three animals from each gender (six total animals) were used for each tissue, and all samples (including standards) were run in duplicate.

\section{ELISA to determine the content of AdipoR1 and AdipoR2}

To assess the total AdipoR1, a competitive ELISA was run as described in the methods section. A standard curve was made using the averaged readings of the 
standards provided by the ELISA kit (Figure 4A) in order to obtain two lines of best fit. The standard curve was split into two separate graphs in order to get the most accurate line of best fit. Using the lines of best fit, the total amount of AdipoR1 and the standard error were calculated (Figure 4B). A t-test was run comparing liver to heart, liver to gastrocnemius, and gastrocnemius to heart, with the two-tailed pvalues listed in Figure 4C after the ANOVA indicated there were significant differences $(p<0.008)$ between the groups. A bar graph (Figure 4D) was compiled using that data and anything with a p-value of less than 0.05 was marked as significantly different. I found that there was a significant difference in the amount of AdipoR1 in the liver compared to the other tissue homogenates; however there were no significant difference found among the other tissues. A Bonferroni correction was done and it was found that the only significant difference that holds up to the new $\alpha$-value $(\alpha \leq 0.008)$ was the liver vs. soleus levels of AdipoR2. A total of ten animals were used (five of each gender) for each tissue type, and all samples (including standards) were run in duplicate. 
A

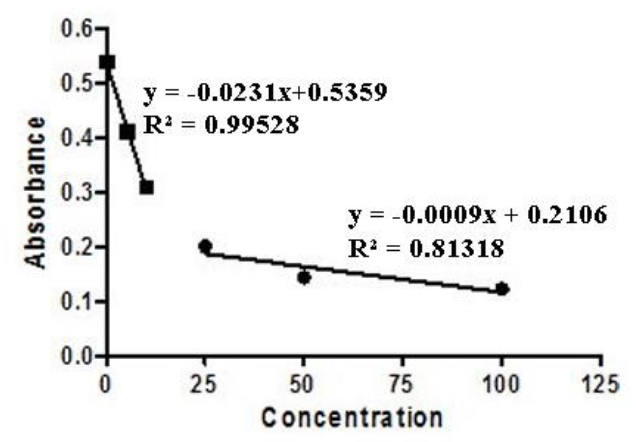

B

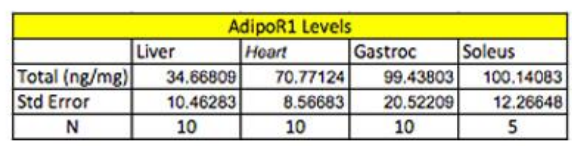

$\mathrm{C}$

\begin{tabular}{|l|r|}
\hline \multicolumn{2}{|c|}{ AdipoR1 p-Value } \\
\hline Liver vs. Heart & 0.01562 \\
\hline Liver vs. Gastroc & 0.01154 \\
\hline Liver vs. Soleus & 0.00143 \\
\hline Heart vs. Soleus & 0.06317 \\
\hline Gastroc vs. Heart & 0.2137 \\
\hline Gastroc vs. Soleus & 0.98062 \\
\hline
\end{tabular}

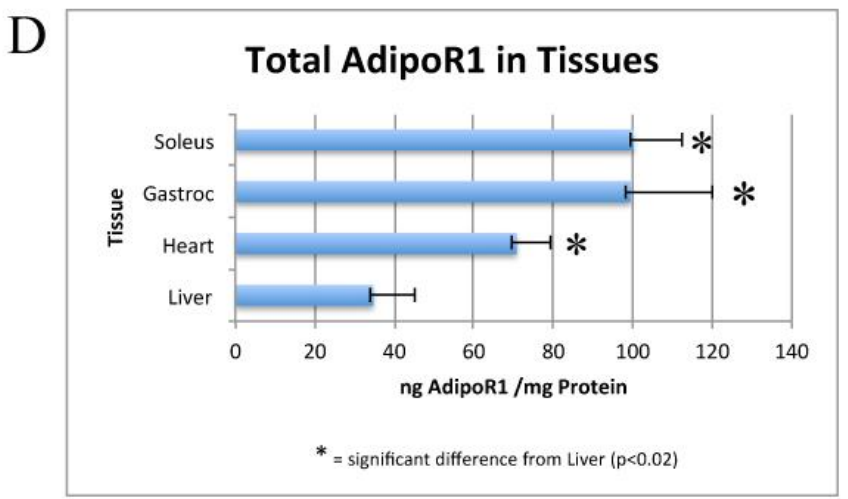

Figure 4: Total AdipoR1 in tissue homogenates. A) The standard curve generated from standards supplied with the ELISA kit. B) The average amount of total AdipoR1 for each of the tissue types accompanied by standard error. C) The p-values from the t-tests comparing the liver to the heart, the liver to the gastrocnemius (gastroc), the liver to the soleus, the heart to the soleus, gastrocnemius to the heart, and gastrocnemius to the soleus. D) A bar graph showing total AdipoR1 levels with significant differences marked. There were a total of ten animals used (five of each gender) for each tissue type, and all samples (including standards) were run in duplicate.

To assess the total AdipoR2 a competitive ELISA was used as previously described above. A standard curve was constructed using the averaged readings of the standards provided by the ELISA kit (Figure 5A) in order to obtain a line of best fit. The total amount of AdipoR2 was found (Figure 5B) and three separate t-tests 
run to compare the liver to heart, the liver to gastrocnemius, and gastrocnemius to heart (Figure 5C) after ANOVA indicated there were significant differences $(p<0.003)$ between the groups. These data were then graphed and any comparison with a p-value less than 0.05 were marked as significantly different (Figure 5D). A significant difference was found between the levels of AdipoR2 in the liver to all other tissues; however there was no significant difference between the other tissues. A Bonferroni correction was performed and all significant differences found for AdipoR2 held true with the new $\alpha$-value $(\alpha \leq 0.0167)$. All tissues were run from six different animals (three of each gender) and all samples (including standards) were done in duplicate. The totals for both AdipoR1 and AdipoR2 were compiled into a single graph (Figure 6) and the significant differences indicated. The totals for AdipoR1 and AdipoR2 for each tissue were tested for significant difference, but only the heart showed significant difference at $p<0.007$. A Bonferroni correction was performed and the significant difference between the receptor levels for the heart held true at the new alpha value of $\alpha \leq 0.0167$. 


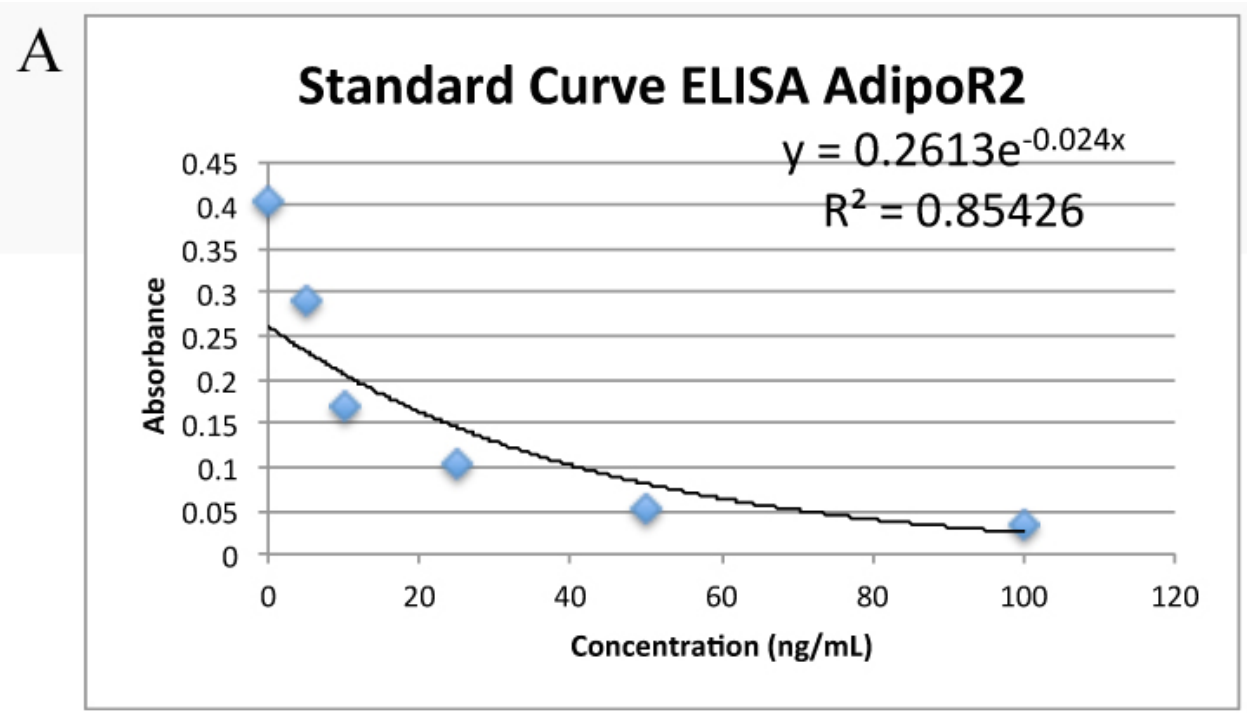

\begin{tabular}{|c|c|c|c|}
\hline \multicolumn{4}{|c|}{ AdipoR2 Levels } \\
\cline { 2 - 5 } & \multicolumn{4}{|c|}{ Liver } & Heart & Gastroc \\
\hline Total $(\mathrm{ng} / \mathrm{mg})$ & 14.79253 & 30.03624 & 50.71138 \\
\hline Std error & 1.90558 & 2.23983 & 9.19922 \\
\hline $\mathrm{N}$ & 6 & 6 & 6 \\
\hline
\end{tabular}
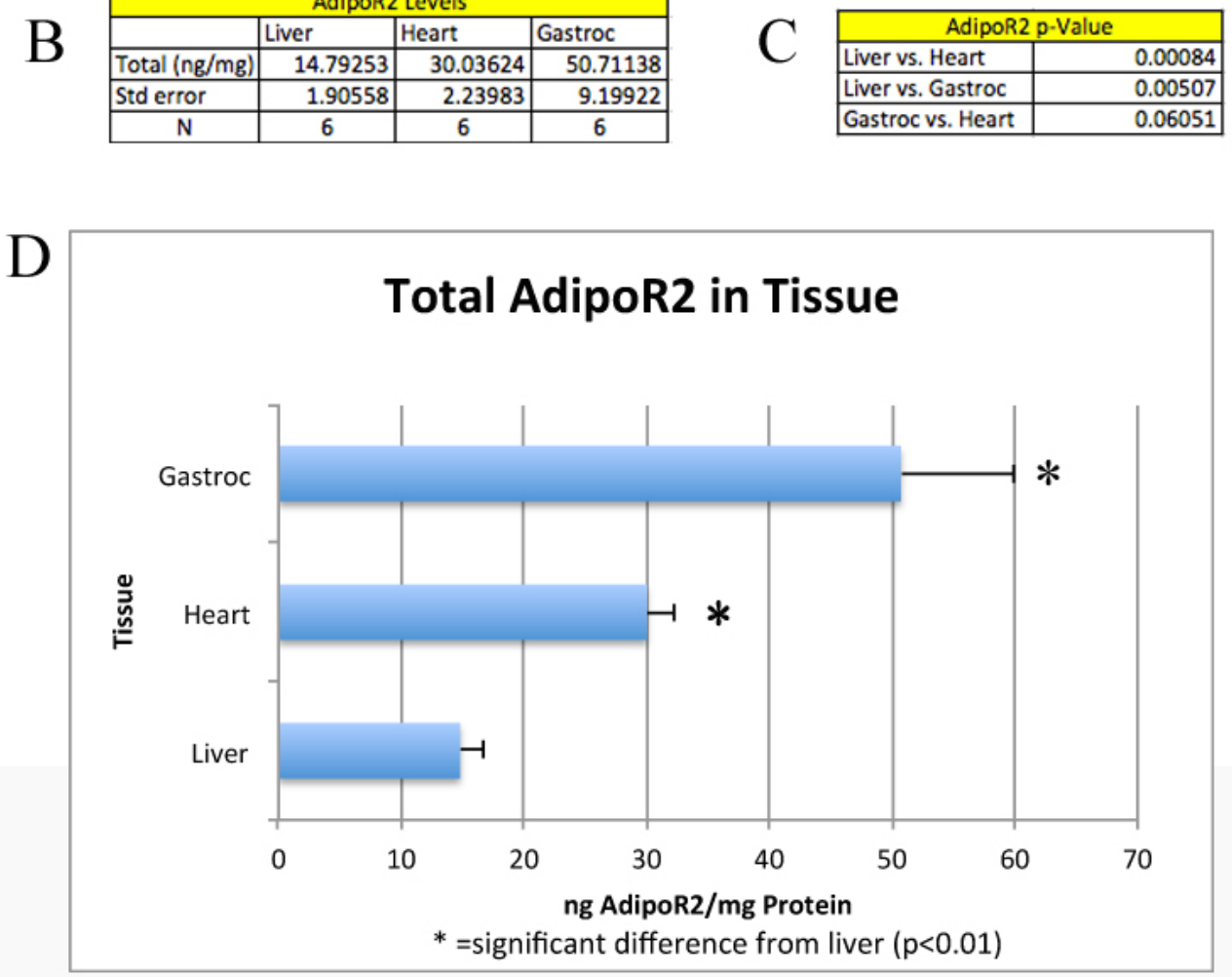

Figure 5: Total AdipoR2 in tissue homogenates. A) The standard curve generated using the standards provided by the ELISA kit. B) The average AdipoR2 for each tissue type accompanied by the standard error. C) The p-values from the t-tests comparing the liver to the heart, the liver to the gastrocnemius, and the gastrocnemius to the heart. D) A bar graph showing the total AdipoR2 for each tissue type with significant differences marked. There 
were a total of six animals used (three of each gender) for each tissue type, and all samples (including standards) were run in duplicate.

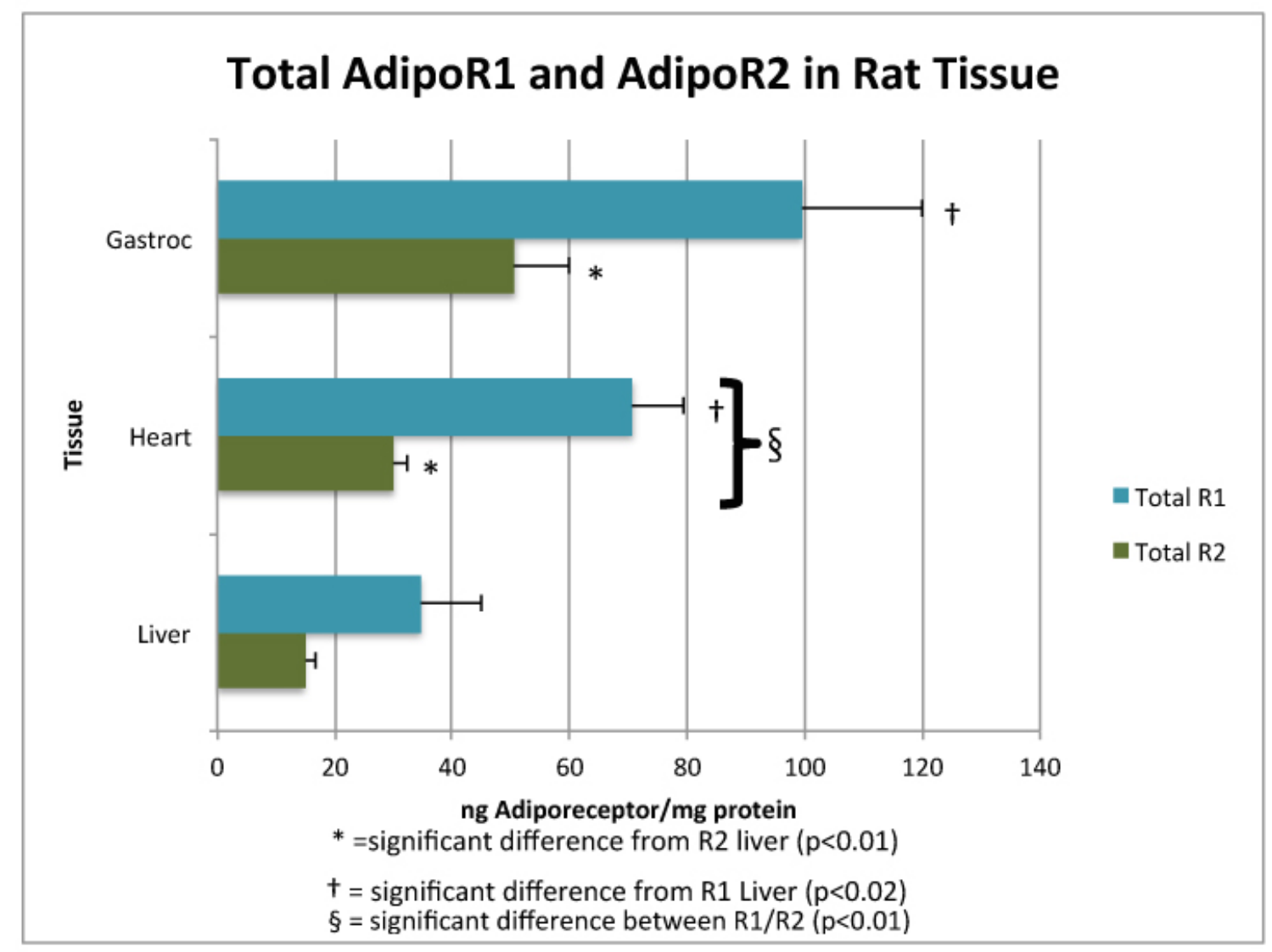

Figure 6: Total AdipoR1 and AdipoR2. Significant difference within the individual receptor levels for different tissue has been marked.

\section{Tissue content of AdipoR1 and AdipoR2 by sex}

The data from both the AdipoR1 and AdipoR2 ELISAs were expressed by gender for the tissue types. The average amount of receptor for each gender and standard error was calculated for each tissue homogenate (Figure 7B/D). T-tests were run between each gender for all the tissue homogenates and the p-values recorded (Figure 7C/E). The results were then compiled into a graph (Figure 7A) 
and the significant differences $(\mathrm{p}<0.05)$ between genders marked. I found that there was significant difference between the male and female liver levels for both receptors. There was also a significant different between the female levels of adipoR1 and the male levels; however, there was no significant difference between the male and female levels of adipoR2. There was also no significant difference between the male and female receptor levels for either receptor. A Bonferroni correction was performed and all significant differences found for AdipoR1/R2 between the sexes held true with the new $\alpha$-value $(\alpha \leq 0.0167)$. For the AdipoR1 samples, five different animals of each gender were used for each tissue type and they were run in duplicate. For the AdipoR2 samples, three different animals for each gender were used for each tissue type and these were also run in duplicate. 


\section{A}

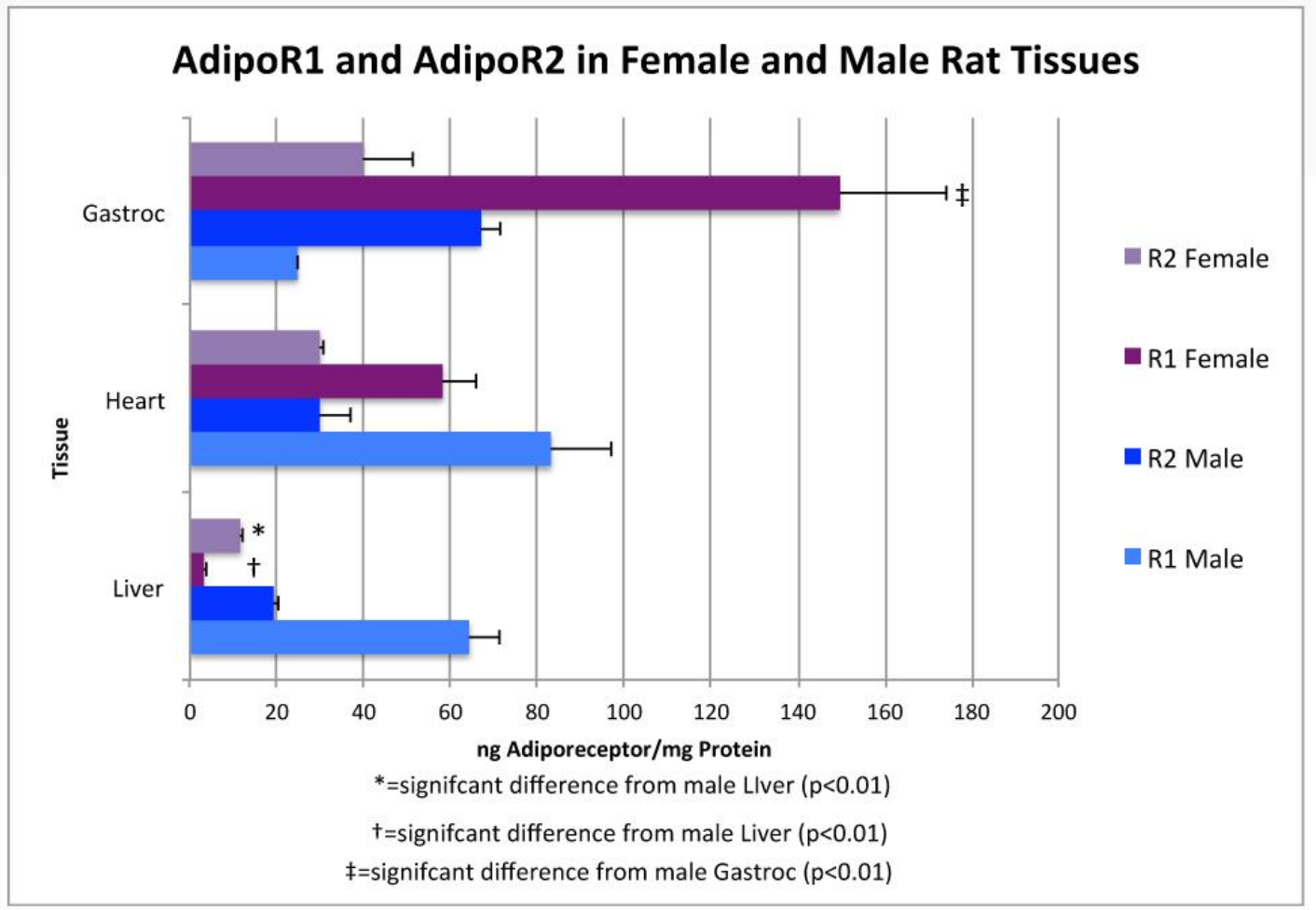

B

\begin{tabular}{|l|c|c|r|}
\hline \multicolumn{4}{|c|}{ Male/Female AdipoR1 } \\
\hline & Liver & Heart & Gastroc \\
\hline R1 Male & 64.42632 & 83.24274 & 24.77444 \\
\hline Std Error & 6.84201 & 13.90209 & 0.23857 \\
\hline R1 Female & 3.03397 & 58.29974 & 149.5468 \\
\hline Std Error & 0.95081 & 7.69548 & 24.69692 \\
\hline \multicolumn{1}{|c|}{$\mathrm{N}$} & $\mathbf{5}$ & $\mathbf{5}$ & 5 \\
\hline
\end{tabular}

$\mathrm{D}$

\begin{tabular}{|l|r|r|r|}
\hline \multicolumn{4}{|c|}{ Male/Female AdipoR2 } \\
\hline R2 Male & 19.35249 & 29.97569 & 66.89533 \\
\hline Std Error & 1.00907 & 7.0013 & 4.76846 \\
\hline R2 Female & 11.75256 & 30.07661 & 39.92208 \\
\hline Std Error & 0.46152 & 0.61758 & 11.35758 \\
\hline N & 3 & 3 & 3 \\
\hline
\end{tabular}

C

\begin{tabular}{|l|c|}
\hline \multicolumn{2}{|c|}{ Male vs. Female AdipoR1 p-Values } \\
\hline Liver & 0.00003 \\
\hline Heart & 0.15512 \\
\hline Gastroc & 0.00416 \\
\hline
\end{tabular}

$\mathrm{E}$

\begin{tabular}{|l|c|}
\hline \multicolumn{2}{|c|}{ Male vs. Female AdipoR2 $p$-Values } \\
\hline Liver & 0.0042 \\
\hline Heart & 0.98595 \\
\hline Gastroc & 0.17176 \\
\hline
\end{tabular}

Figure 7: Adiponectin receptor levels by sex. A) A bar graph showing the levels of the two adiponectin receptors for each gender related to tissue type. Significant difference between the genders has been marked. B) The average amount of AdipoR1 for male and female tissue as well as the standard error for each gender. C) The p-values of the t-tests comparing the male and female AdipoR1 levels for each tissue. D) The average amount of AdipoR2 for male and female tissue as well as the standard error for each gender. E) The pvalues of the t-tests comparing the male and female AdipoR2 levels for each tissue. All 
samples were run in duplicate with at least three animals of each gender used for each tissue type.

\section{Discussion}

As noted in the literature $(1,3,4,5,7,10,11)$, the present study has confirmed a higher AdipoR1 level in muscles compared to liver. Furthermore, this study demonstrated that there was no significant difference between the total AdipoR1 levels between muscle types, as can be seen in Figure 4. Since AdipoR1 is best known for its ability to increase mitochondrial function and thereby improve metabolic capacity as well as glucose uptake, it follows that the levels would be higher in the muscles than the liver since the muscles consume glucose as their main form of energy. This makes AdipoR1 a potential target for pharmaceutical therapies that could potentially increase the amount of AdipoR1 found in the muscle cell membranes or increase the sensitivity of the receptor to adiponectin in an effort to allay the disease states associated with diabetes and obesity.

This study also found that the gastrocnemius and liver are significantly different between the genders (Figure 7). The livers from male rats have a significantly higher amount of AdipoR1 than those from female, and the female gastroc samples have a significantly higher amount of AdipoR1 compared to the males (Figure 7). These findings may correlate to previous results from this lab that suggested that there was a higher level of lower molecular weight (LMW) adiponectin produced by female muscles compared to male muscles (data not 
shown). Because the AdipoR1 receptor has been reported to have a higher affinity for globular adiponectin (LMW), this may reveal a physiological relevance.

Contrary to the literature $(1,3,4,5,7,10,11)$, the total level of AdipoR2 found in the liver was significantly lower than that of the muscle tissues tested (Figure 5). Previous studies $(1,3,4$ have found that the levels of AdipoR2 are significantly higher in the liver than in the muscles. The ability of AdipoR2 to increase fatty acid oxidation in the liver (19) is important in re-sensitizing the liver to insulin. If there is an increase in oxidative stress in the liver, gluconeogenesis will increase in the liver and glucose uptake will decrease glucose uptake in the liver and skeletal muscles (23). This effect of adipoR2 makes is a potential target for pharmaceutical therapy as well, but due to the lowered levels found in this study, it may pose difficulties for further therapeutic testing. The lowered amount in the rats used for this study could indicate that the rats were under oxidative stress before death. To clarify the present results for AdipoR2, further studies such as immunoblotting should be performed to confirm the ELISA results.

There was a significant difference in AdipoR2 levels found between the genders for the liver, but there were no significant differences between the genders for the other tissue samples (Figure 7). The differences between the genders for the liver may have a physiological relevance as well, but further study is needed.

The levels of AdipoR1/R2 in the liver were significantly different between the females as well as between the males. The levels of AdipoR1/R2 in the heart were significantly different between the females, but not between the males. Finally, the levels of AdipoR1/R2 in the gastrocnemius were significantly different between the 
females, but not between the males. A sexual dimorphism has been reported in the levels of secreted adiponectin (17) in humans and rodents, so it is possible that there is a similar mechanism that affects the amount of AdipoR inserted into the cellular membrane.

Based on the data gathered so far, the rat makes a comparable model to mouse and human when it comes to AdipoR1 receptors. However, my data indicated that it might not make a good model for AdipoR2 receptors since AdipoR2 receptors in humans and mice are reported to be higher in the liver than in muscle $(1,3,4,5,7,10,11)$. Further studies are required to confirm the perceived inconsistencies for AdipoR2 from the current findings. This is needed in order to determine if rats are different from mice and humans for this receptor.

\section{Acknowledgments}

I would like to offer my gratitude to Dr. Joseph Steffen, who not only provided me with the opportunity to perform this research, but who also provided invaluable guidance during this journey. I would also like to thank Amber Hussain and Christina Seibert who helped with tissue homogenization, and Jacob Edmisson who provided technical support. This project was supported by funds from the Office of Executive Vice President for Research and Innovation. 


\section{Bibliography}

1. Lustig, Y., Hemi, R., \& Kanety, H. (2012). Regulation and Function of Adiponectin Receptors in Skeletal Muscle. Vitamins and Hormones, 90, 95123.

2. Hui, X., Lam, K. S., Vanhoutte, P., \& Xu, A. (2011). Adipnectin and cardiovascular health. British Journal of Pharmacology, 165, 574-590.

3. Kadowaki, T., \& Yamauchi, T. (2005). Adiponectin and Adiponectin Receptors. Endocrine Reviews, 26 (3), 439-451.

4. Yamauchi, T., Iwabu, M., Okada-Iwabu, M., \& Kadowaki, T. (2013).

Adiponectin receptors: A review of thier structure, function, and how they work. Elsevier, 28, 15-23.

5. Nannipieri, M., Bonotti, A., Anselmino, M., Cecchetti, F., Madec, S., Manicini, E., et al. (2007). Pattern of expression of adiponectin receptors in human adipose tissue depots and its relation to the metabolic state. International Journal of Obesity, 31, 1843-1848.

6. Lee, S., \& Kwak, H.-B. (2014). Effects of interventions on adiponectin and adiponectin receptors. Journal of Exercise Rehabilitation, 10, 60-68.

7. Yamauchi, T., \& Kadowaki, T. (2013). Adiponectin Receptor as a Key Player in Healthy Longevity and Obesity-Related Disease. Elsevier, 17, 185-196.

8. Lin, T., Qui, Y., Mohan, R., Li, Q., \& Lei, B. (2013). Expression of adiponectin and its receptors in type 1 diabetes mellitus in human and mouse retinas. Molecular Vision, 19, 1769-1778.

9. Ma, Y., Liu, Y., Liu, S., Qu, Y., Wang, R., Xia, C., et al. (2011). Dynamic alteration 
of adiponectin/adiponectin receptor expression and its impact on myocardial ischemia/reperfusion in type 1 diabetic mice. AJP Endocrinology Metabolism, 301, 447-455.

10. Bullen, J. W., Bluher, S., Kelesidis, T., \& Mantzoros, C. (2006). Regulation of adiponectin and its receptors in response to development of diet induced obesity in mice. AJP Endocrinology Metabolism, 292, 1079-1086.

11. Dadson, K., Liu, Y., \& Sweeney, G. (2011). Adiponectin action: a combination of endocrine and autocrine/paracrine effects. Frontiers in Endocrinology, 2, 114.

12. Kovacova, Z., Tencerova, M., Roussel, B., Wedellova, Z., Rossmeislova, L., Langin, D., et al. (2012). The impact of obesity on secretion of adiponectin multimeric isoforms differs in visceral and subcutaneous adipose tissue. International Journal of Obesity, 36, 1360-1365.

13. Guo, Z., Zhihong, Q., Zhang, R., Li, J., \& Yin, Y. (2012). Effect of rosiglitazone on the expression of cardiac adiponectin receptors and NADPH oxidase in type 2 diabetic rats. European Journal of Pharmacology, 685, 116-125.

14. Liu, M., \& Liu, F. (2010). Transcriptional and post-translational regulation of adiponectin. BJ Metabolism, 425, 41-52.

15. Ogden, C. L., \& et al. (2014). Prevalence of Childhood and Adult Obesity in the United States, 2011-2012. JAMA, 311 (8), 806-814.

16. Schacterle, G R, Polluck, R L. (1973). A simplified method for the quantitative assay of small amounts of protein in biologic material. Analytical Biochemistry, 51, 645-655. 
17. Wang, Y., Lam, K., Yau, M.-h., \&Xu, A. (2008). Post-translational modifications of adiponectin: Mechanism and functional implications. Biochemistry Journal, 623-633.

18. Bogan, J. S., Lodish, H. F. (1999). Two compartments for insulin stimulated exocytosis in 2T2-Li adipocytes defined by endogenous ACRP30 and GLUT4. Journal of Cellular Biology, 146, 609-620.

19. Kadowaki, T., Yamauchi, T., Kubato, N., Hara, K., Ueki, K., \& Tobe, K. (2006). Adiponectin and adiponectin receptors in insulin resistance, diabetes, and the metabolic syndrome. The Journal of Clinical Investigation , 116 (7), 17841792.

20. Yamauchi, T., et all. (2007). Targeted disruption of AdipoR1 and AdipoR2 causes abrogation of adiponectin binding and metabolic actions. Nature Medicine, 13 (3), 332-339.

21. Ujiie, H., et all. (2006) Identification of Amino-Terminal Region of Adiponectin as a Physiologically Functional Domain. Journal of Cellular Biochemistry, 98, 194-207.

22. Goldstein, B. J., Scalia, R. G., \& Ma, X. L. (2009). Protective vascular and myocardial effects of adiponectin. Nature Clinical Practice. Cardiovascular Medicine, 6(1), 27-35.

23. Okada-Iwabu, M., Iwabu, M., Ueki, K., Yamauchi, T., \& Kadowaki, T. (2015). Perspective of Small-Molecule AdipoR Agonist for Type 2 Diabetes and Short Life in Obesity. Diabetes \& Metabolism Journal, 39(5), 363-372. 